MATHEMATICS OF COMPUTATION

Volume 73, Number 247, Pages 1443-1458

S $0025-5718(03) 01592-8$

Article electronically published on July 29, 2003

\title{
BOUNDS FOR COMPUTING THE TAME KERNEL
}

\author{
RICHARD P. GROENEWEGEN
}

\begin{abstract}
The tame kernel of the $K_{2}$ of a number field $F$ is the kernel of some explicit map $K_{2} F \rightarrow \bigoplus k_{v}^{*}$, where the product runs over all finite primes $v$ of $F$ and $k_{v}$ is the residue class field at $v$. When $S$ is a set of primes of $F$, containing the infinite ones, we can consider the $S$-unit group $U_{S}$ of $F$. Then $U_{S} \otimes U_{S}$ has a natural image in $K_{2} F$. The tame kernel is contained in this image if $S$ contains all finite primes of $F$ up to some bound. This is a theorem due to Bass and Tate. An explicit bound for imaginary quadratic fields was given by Browkin. In this article we give a bound, valid for any number field, that is smaller than Browkin's bound in the imaginary quadratic case and has better asymptotics. A simplified version of this bound says that we only have to include in $S$ all primes with norm up to $4|\Delta|^{3 / 2}$, where $\Delta$ is the discriminant of $F$. Using this bound, one can find explicit generators for the tame kernel, and a "long enough" search would also yield all relations. Unfortunately, we have no explicit formula to describe what "long enough" means. However, using theorems from Keune, we can show that the tame kernel is computable.
\end{abstract}

\section{INTRODUCTION AND STATEMENT OF THE MAIN THEOREM}

The explicit determination of the tame kernel of a number field is similar to the determination of class groups. As a consequence of a suitable Minkowski bound, the computations are restricted to a finite set of primes with small norms. Using these primes, one searches for relations until finally one can prove, or at least conjecture, that the found generators and relations yield the whole group. It is useful to have a small upper bound on the norm of the primes used.

In order to make the above discussion more explicit, we give some definitions. Let $F$ be a number field with ring of integers $\mathcal{O}$ and discriminant $\Delta$. We define the group $K_{2} F$ as

$$
K_{2} F=\left(F^{*} \otimes F^{*}\right) /\left\langle a \otimes b: a, b \in F^{*}, a+b=1\right\rangle .
$$

All tensor products in this article are taken over $\mathbf{Z}$. The class belonging to $a \otimes b$ is written as $\{a, b\}$ and the group operation is written multiplicatively. There is a more general definition of the $K_{2}$ of a ring, as a group of nonobvious matrix relations, but we do not need it here. It can be found in [12.

For $v: F \rightarrow \mathbf{Z} \cup\{\infty\}$ a nonarchimedean valuation corresponding to the prime ideal $\mathfrak{p}_{v}$ of $\mathcal{O}$ with residue class field $k_{v}=\mathcal{O} / \mathfrak{p}_{v}$, we define a map $t_{v}: K_{2} F \rightarrow k_{v}^{*}$ by

$$
\{a, b\} \longmapsto(-1)^{v(a) v(b)} \frac{a^{v(b)}}{b^{v(a)}} \bmod \mathfrak{p}_{v} .
$$

Received by the editor April 18, 2002 and, in revised form, December 6, 2002.

2000 Mathematics Subject Classification. Primary 11R70; Secondary 11Y40, 19C20.

$K e y$ words and phrases. $K$-theory, tame kernel, calculations, $S$-units.

(C)2003 American Mathematical Society 
We will often refer to valuations as primes. The tame kernel is defined as the kernel of the map

$$
\left(t_{v}\right)_{v<\infty}: K_{2} F \longrightarrow \bigoplus_{v<\infty} k_{v}^{*}
$$

Using the more general definition of the $K_{2}$, one can show that the tame kernel is equal to $K_{2} \mathcal{O}$ (see [13, $\S 5$, corollary to Theorem 5]). It is a finite group (see [6]) and when we talk about determining it explicitly, we mean giving a finite number of generators and relations for the group.

The determination of the tame kernel is made manageable with the use of a filtration of $K_{2} F$. For any set $S$ of primes of $F$ that contains the set $S_{\infty}$ of infinite primes, we define $U_{S}$ as the group of $S$-units of $F$, i.e., $U_{S}=\left\{x \in F^{*}: v(x)=\right.$ 0 for all $v \notin S\}$. For every positive integer $m$, we write

$$
U_{m}=U_{S_{m}}, \quad \text { where } \quad S_{m}=S_{\infty} \cup\{\text { all finite primes } v \text { with } N v \leq m\} .
$$

Here, the norm $N v$ denotes the order $\# k_{v}$ of the residue class field. Now we can define

$$
K^{(m)}=\left(U_{m} \otimes U_{m}\right) /\left\langle a \otimes b: a, b \in U_{m}, a+b=1 \text { or } a+b=0\right\rangle .
$$

In $K_{2} F$, we have $\{-a, a\}=1$ for all $a \in F^{*}$. For $a \neq 1$ we see this from the computation $\{-a, a\}=\{-a, a\}\left\{1-a^{-1}, a^{-1}\right\}^{-1}=\{-a+1, a\}=1$. That is why there is a natural map $K^{(m)} \rightarrow K_{2} F$. We write $K^{m}$ for the image of $K^{(m)}$ in $K_{2} F$. The maps $K^{m-1} \rightarrow K^{m}$ are injective, whereas the maps $K^{(m-1)} \rightarrow K^{(m)}$ may not be. For every $m$ we have an "approximation" $K_{2}^{(m)} \mathcal{O}$ of the tame kernel, defined by

$$
K_{2}^{(m)} \mathcal{O}=\operatorname{ker}\left[K^{(m)} \longrightarrow \bigoplus_{N v \leq m} k_{v}^{*}\right]
$$

where the direct sum is taken over all finite primes with norm up to $m$. The groups $K_{2}^{(m)} \mathcal{O}$ have two important virtues. As we will see, $K_{2}^{(m)} \mathcal{O}$ is computable as a function of $m$ and $F$, meaning that there is an algorithm that, given $m$ and $F$ in some explicit way, computes the group in a finite number of steps. Furthermore, when $m$ is large enough, the group $K_{2}^{(m)} \mathcal{O}$ is equal to the tame kernel.

We can now state a simplified version of the main theorem of this article.

Theorem 1. For every number field $F$, there are constants $c_{F}, c_{F}^{\prime}$ such that

(1) For all $m>c_{F}$, the map $K^{(m)} / \mathrm{im} K^{(m-1)} \rightarrow \bigoplus_{N v=m} k_{v}^{*}$ induced by the maps $t_{v}$ is an isomorphism. The direct sum is taken over all finite primes with norm equal to $m$.

(2) For all $m>c_{F}^{\prime}$, the map $K^{(m)} \rightarrow K^{m}$ is an isomorphism.

For $m>c_{F}$, the image of $K_{2}^{(m)} \mathcal{O}$ in $K_{2} F$ is equal to the tame kernel. For $m>$ $\max \left\{c_{F}, c_{F}^{\prime}\right\}$, the natural map $K_{2}^{(m)} \mathcal{O} \rightarrow K_{2} \mathcal{O}$ is an isomorphism. We can take $c_{F}=4|\Delta|^{3 / 2}$. The group $K_{2}^{(m)} \mathcal{O}$ is computable as a function of $m$ and $F$.

This theorem is a consequence of Theorem 15, in which we give a different $c_{F}$, which is smaller by Proposition 21. Anyone who wants to use our theorem for calculations should use the results in Section 8 .

Part 1 of the theorem, without an explicit $c_{F}$, is a reformulation of Theorem II.3.1 in an article of Bass and Tate [1]. Careful inspection of their arguments led to explicit bounds, and for imaginary quadratic number fields the best bound so far was given by Browkin [2]. He proves that we can take $c_{F}=2^{6} \pi^{-10 / 3}|\Delta|^{5 / 3}$ 
when $F$ is imaginary quadratic and $|\Delta|$ is at least 15 . Clearly, the bound in our theorem above is asymptotically better. In Section 8 , we work out what the bound in Theorem [15] amounts to in the imaginary quadratic case. It turns out our bound outperforms Browkin's bound for small discriminants also. Our results are compared to those of Browkin in Section 8 .

One can find explicit generators for the tame kernel by finding generators for $K_{2}^{(m)} \mathcal{O}$, where we have $m>c_{F}$. Unfortunately, we have no explicit formula for $c_{F}^{\prime}$. However, using theorems from Keune 8 that allow us to determine the order of the $p$-primary part of the tame kernel, we can compute the tame kernel and hence also $c_{F}^{\prime}$.

Theorem 2. The smallest feasible value of $c_{F}^{\prime}$ and the tame kernel $K_{2} \mathcal{O}$ are computable as a function of the number field $F$.

This theorem is a combination of Theorem 19] and Theorem [20 from Section 8 Basically, we take an $m>c_{F}$ that is large enough such that the kernel of the map $K^{(m)} \rightarrow \bigoplus_{N v \leq m} k_{v}^{*}$ is finite. Then the tame kernel is a quotient of this kernel, which gives a bound on the order of the tame kernel and allows us to use Keune's theorems.

The first calculations of tame kernels were done by Tate, who computed the tame kernels for the six imaginary quadratic fields with $|\Delta| \leq 15$ in the appendix of [1]. Recent, but currently unpublished, calculations using the bound in this article are done by K. Belabas and H. Gangl. They have independently been using Keune's theorems to prove correctness of their results. Some of their results are stated in the appendix of [2].

While proving the main theorem, we need to find "small" generators of $S$-unit groups. In Section 5, we prove the following theorem.

Theorem 3. Let $s$ be the number of complex primes of $F$ and let $T$ be a finite set of primes of $F$ containing $S_{\infty}$ and all finite primes with norm at most $(2 / \pi)^{s}|\Delta|^{1 / 2}$. Write $m_{T}=\max \left(\{1\} \cup\left\{N v: v \in T-S_{\infty}\right\}\right)$. Then $U_{T}$ is generated by the set of all $a \in \mathcal{O} \cap U_{T}$ with $\left|a_{v}\right|_{v} \leq(2 / \pi)^{2 s}|\Delta| m_{T}$ for all $v \in S_{\infty}$.

Remark. By saying the tame kernel $K_{2} \mathcal{O}$ is computable as a function of $F$, we mean that the function that sends a number field $F$ to the tame kernel $K_{2} \mathcal{O}$ is computable. More generally, we say $f(x)$ is computable as a function of $x$, when we actually mean that $f$ is computable. Furthermore, our statements about computability depend on how the input of the function is represented. We feel it is not necessary to be very precise about this, because any noncontrived representation usually works well. For instance, a natural number can be represented as a finite bit-string, giving the binary representation. A number field is represented by a generating element and this element itself is represented by a fundamental polynomial with coefficients in $\mathbf{Z}$. For the representation of other objects, we refer to [4, particularly Chapter 4. In [4] one can also find algorithms to compute the maximal order and the class group of a number field.

\section{Notation And outline of the ARTicle}

This section is mainly concerned with setting the notation and explaining the strategy we use to prove the main theorem. At the end of this section, we give an outline of the remainder of the article. 
The setting is the same as in the introduction: we have a number field $F$ with ring of integers $\mathcal{O}$ and discriminant $\Delta$. Let $n=r+2 s$ be the degree of $F$, where $r$ is the number of real primes and $s$ is the number of complex primes. In the introduction we constructed groups $K^{(m)}$ which gave a filtration of $K_{2} F$. Here, we will make the filtration even finer. Let $v_{1}, v_{2}, v_{3}, \ldots$ be an ordering of all the finite primes with nondecreasing norms. Let $v$ be a finite prime. Then there is an index $j$ with $v_{j}=v$. We define the sets $S=S_{\infty} \cup\left\{v_{1}, v_{2}, \ldots, v_{j-1}\right\}$ and $S^{\prime}=S \cup\{v\}$ and we write

$$
\begin{aligned}
U & =U_{S}, & K & =(U \otimes U) /\langle a \otimes b: a, b \in U: a+b=1 \text { or } a+b=0\rangle, \\
U^{\prime} & =U_{S^{\prime}}, & K^{\prime} & =\left(U^{\prime} \otimes U^{\prime}\right) /\left\langle a \otimes b: a, b \in U^{\prime}: a+b=1 \text { or } a+b=0\right\rangle .
\end{aligned}
$$

Hence, $S, S^{\prime}, U, U^{\prime}, K, K^{\prime}$ depend on $v$ and on the numbering of the primes, although this is not visible in the notation. We will prove that under certain conditions on the norm of $v$, the map $K^{\prime} / \mathrm{im} K \rightarrow k_{v}^{*}$ is an isomorphism and we deduce the main theorem from that.

It is crucial for the remainder of this article that the sequence

$$
0 \longrightarrow U \longrightarrow U^{\prime} \stackrel{v}{\longrightarrow} \mathbf{Z} \longrightarrow 0
$$

is exact. It is, however, not true in general that the valuation map is surjective when the norm of $v$ is very small.

Lemma 4. Define

$$
d=\frac{2^{n} \Gamma(n / 2+1)}{(\pi n)^{n / 2}}|\Delta|^{1 / 2}
$$

and let $\rho=\rho_{n}$ be the packing density of an $n$-dimensional sphere. Then the valuation map $U^{\prime} \stackrel{v}{\longrightarrow} \mathbf{Z}$ is surjective as long as $N v>\rho d$. We have $\rho d \geq 1$.

Before we give the proof, we want to give some definitions and explain the constant $\rho$. First we explain packing densities. Let $K_{0}$ be a measurable, bounded subset of $\mathbf{R}^{n}$ with nonempty interior. A packing of $K_{0}$ in $\mathbf{R}^{n}$ is a collection $\mathcal{K}$ of translates of $K_{0}$ such that the interiors do not meet pairwise. Let $C_{r}$ (for $r \in \mathbf{R}_{>0}$ ) be the hypercube in $\mathbf{R}^{n}$ consisting of points with each of the coordinates in absolute value at most $r$. Then we define the density of $\mathcal{K}$ as

$$
\rho_{+}(\mathcal{K})=\limsup _{r \rightarrow \infty}\left(\operatorname{vol}\left(C_{r}\right)^{-1} \sum_{\substack{K_{0}^{\prime} \in \mathcal{K} \\ K_{0}^{\prime} \cap C_{r} \neq \emptyset}} \operatorname{vol}\left(K_{0}^{\prime}\right)\right),
$$

where "vol" is the volume given by the Lebesgue measure on $\mathbf{R}^{n}$. Intuitively, $\rho_{+}(\mathcal{K})$ is the proportion of the entire space covered by the union of the elements in $\mathcal{K}$. The packing density of $K_{0}$ is defined as

$$
\rho\left(K_{0}\right)=\sup _{\mathcal{K}} \rho_{+}(\mathcal{K})
$$

where the supremum is taken over all packings $\mathcal{K}$ of $K_{0}$. In the case $K_{0}$ is an $n$-dimensional sphere, we have

$$
\rho=\rho_{n}=\rho\left(K_{0}\right) \leq \frac{n+2}{2}\left(\frac{1}{\sqrt{2}}\right)^{n} \quad \text { and } \quad \rho_{n}=\pi / \sqrt{12} \quad \text { if } n=2 .
$$

Asymptotically stronger is the Kabatiansky-Levenshtein bound, which says

$$
\rho_{n} \leq 2^{-0.599 n+o(n)} \text { for } n \rightarrow \infty \text {. }
$$


For references and more information about packings, we refer to Rogers [14 and Conway and Sloane [5].

Lemma 5 (Minkowski). Let $L$ be a lattice of $\mathbf{R}^{n}$ of full rank, with determinant $\operatorname{det}(L)$. Let $K_{0}$ be a closed, convex, symmetric subset of $\mathbf{R}^{n}$ such that $\operatorname{vol} K_{0} \geq$ $2^{n} \rho\left(K_{0}\right) \operatorname{det}(L)$. Then there is a nonzero point in $L \cap K_{0}$.

Proof. This is a trivial adaptation from [10, V.3, Theorem 3].

The $\mathbf{R}$-vector space we will be working in is $F_{\mathbf{R}}=F \otimes \mathbf{R}$. The additive group of $F_{\mathbf{R}}$ is naturally equivalent to its own character group by sending $x \in F_{\mathbf{R}}$ to the character $y \mapsto e^{-2 \pi i \operatorname{Tr}(x y)}$, where $\operatorname{Tr}$ denotes the trace from $F_{\mathbf{R}}$ to $\mathbf{R}$. Using this equivalence, we let the measure on $F_{\mathbf{R}}$ be the self-dual measure. This means we take the measure $d x$ such that we have

$$
f(x)=\hat{\hat{f}}(-x)
$$

for continuous complex valued functions $f \in L_{1} F_{\mathbf{R}}$ for which the Fourier transform $\hat{f}(y)=\int f(x) e^{2 \pi i \operatorname{Tr}(x y)} d x$ is also continuous and in $L_{1} F_{\mathbf{R}}$ (see [15]). Consequently, the determinant of $\mathcal{O}$ is equal to $|\Delta|^{1 / 2}$.

In order to apply Minkowski, we need a supply of closed, convex, symmetric sets. We will mainly use balls, cubes and diamonds. We define these shapes in terms of three corresponding metrics. Write $F_{\mathbf{R}}$ as the product

$$
F_{\mathbf{R}}=\prod_{v \in S_{\infty}} F_{v} .
$$

An element $x \in F_{\mathbf{R}}$ has coordinates $x_{v} \in F_{v}$. Each factor in the product has an absolute value $|\cdot|_{v}$ by embedding $F_{v}$ into $\mathbf{C}$. For $t \in \mathbf{R}_{>0}$, we define

balls: $\quad B_{t}=\left\{x \in F_{\mathbf{R}}:\|x\|_{2} \leq t^{1 / n}\right\}$, with $\|x\|_{2}=\left(\frac{1}{n} \sum_{w \in S_{\infty}} n_{w}\left|x_{w}\right|_{w}^{2}\right)^{1 / 2}$, cubes: $\quad C_{t}=\left\{x \in F_{\mathbf{R}}:\|x\|_{\infty} \leq t^{1 / n}\right\}$, with $\|x\|_{\infty}=\max _{w \in S_{\infty}}\left|x_{w}\right|_{w}$, diamonds: $D_{t}=\left\{x \in F_{\mathbf{R}}:\|x\|_{1} \leq t^{1 / n}\right\}$, with $\|x\|_{1}=\frac{1}{n} \sum_{w \in S_{\infty}} n_{w}\left|x_{w}\right|_{w}$,

where $n_{w}$ is equal to the degree $\left[K_{w}: \mathbf{R}\right]$.

The basic properties that we use of these sets are listed in the lemma below.

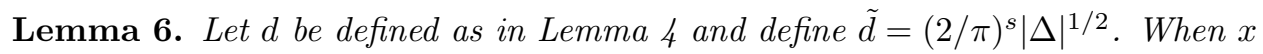
is an element of $F$, we write $N(x)$ for the absolute value of the norm of $x$ to $\mathbf{Q}$. For $t \in \mathbf{R}_{>0}$, the norm $N(x)$ of an element $x$ in either $B_{t}, C_{t}$ or $D_{t}$ is at most $t$. The volumes of $B_{d t}$ and $C_{\tilde{d} t}$ are given by

$$
\operatorname{vol} B_{d t}=\operatorname{vol} C_{\tilde{d} t}=2^{n}|\Delta|^{1 / 2} t
$$

For $t, t^{\prime} \in \mathbf{R}_{>0}$, we have

$$
B_{t} B_{t^{\prime}} \subset D_{t t^{\prime}}, \quad B_{t} C_{t^{\prime}} \subset B_{t t^{\prime}} .
$$

Remarks and proof. The volume of an $n$-dimensional sphere with radius 1 in $\mathbf{R}^{n}$ is

$$
\frac{\pi^{n / 2}}{\Gamma(n / 2+1)} \text {. }
$$

We have $\Gamma(1 / 2)=\sqrt{\pi}$ and $\Gamma$ satisfies the functional relation $\Gamma(x)=(x-1) \Gamma(x-1)$. The measure on $F_{\mathbf{R}}$ induced by $\|\cdot\|_{2}$ and the canonical measure on $F_{\mathbf{R}}$ we use to calculate volumes differ by the factor $n^{n / 2}$. From this, the formula for the volume of $B_{d t}$ follows. 
The statement that norms of elements in $B_{t}, C_{t}$ and $D_{t}$ are bounded by $t$ is an easy application of the inequality of the means. By $B_{t} B_{t^{\prime}}$ we mean the set $\{x y$ : $\left.x \in B_{t}, y \in B_{t^{\prime}}\right\}$ and $B_{t} B_{t^{\prime}} \subset D_{t t^{\prime}}$ follows from the Cauchy-Schwarz inequality.

We are now ready to prove Lemma 4 ,

Proof of Lemma 4 Let $d$ and $\rho$ be defined as in the lemma. We have

$$
\operatorname{vol} B_{d \rho}=2^{n} \rho|\Delta|^{1 / 2}=2^{n} \rho \operatorname{det}(\mathcal{O}) .
$$

Hence, by Minkowski there is a nonzero point in $\mathcal{O} \cap B_{d \rho}$. The norm of this element is at least 1 because it is in $\mathcal{O}$, and it is at most $d \rho$ because it is in $B_{d \rho}$. Hence, $d \rho$ is at least 1.

Now let $\mathfrak{p}=\mathfrak{p}_{v}$ be the prime ideal corresponding to $v$ and set $t=d \rho N v$. We have vol $B_{t}=2^{n} \rho|\Delta|^{1 / 2} N v=2^{n} \rho \operatorname{det}(\mathfrak{p})$ and therefore there exists a nonzero point $\pi$ in $\mathfrak{p} \cap B_{t}$, for which we clearly have $v(\pi) \geq 1$. Because $\pi$ is an integral element, $v(\pi)$ can only be greater than 1 if $N \pi$ is at least $(N v)^{2}$. But we have $N \pi \leq t=$ $d \rho N v<(N v)^{2}$. Hence, we have $v(\pi)=1$ and $\pi \in U^{\prime}$, which proves the surjectivity of the valuation map.

We adopt the notation for $d$ and $\rho$ from the lemma and we will make the following assumption:

$$
\text { Assumption 1: } N v>\rho d \text {. }
$$

With this assumption, the set

$$
\Pi=\left\{\pi \in U^{\prime}: v(\pi)=1\right\}
$$

is not empty. Let $\pi$ be any element from $\Pi$ and consider the map

$$
U \longrightarrow K^{\prime} / \operatorname{im} K, \quad \text { given by } u \longmapsto\{u, \pi\} .
$$

By abuse of notation, $\{u, \pi\}$ denotes both an element in $K^{\prime}$ and in the quotient.

Lemma 7. The map $U \rightarrow K^{\prime} / \operatorname{im} K$ does not depend on the choice of $\pi \in \Pi$. The map is surjective and the kernel contains $U \cap(1+\Pi)$.

Proof. For any $\pi^{\prime} \in \Pi$ we can write $\pi^{\prime}=u^{\prime} \pi$ for some $u^{\prime} \in U$ and we have

$$
\left\{u, \pi^{\prime}\right\} /\{u, \pi\}=\left\{u, u^{\prime}\right\} \in \operatorname{im} K .
$$

Hence, the map does not depend on $\pi$. To see that the map is surjective, first notice that $K^{\prime} / \operatorname{im} K$ is certainly generated by elements of the form $\{u, \pi\},\{\pi, u\}$, $\{\pi, \pi\}$, for $u \in U$. It follows from the definitions that $\{\cdot, \cdot\}$ is anti-symmetric: for all $a, b \in U^{\prime}$, we have

$$
\{a, b\}\{b, a\}=\{a, b\}\{-b, b\}\{b, a\}\{-a, a\}=\{-a b, a b\}=1 .
$$

So, we can omit the generators $\{\pi, u\}$ in favor of $\{u, \pi\}$. We rewrite

$$
\{\pi, \pi\}=\{\pi, \pi\} /\{-\pi, \pi\}=\{-1, \pi\},
$$

so this element is also covered by elements of the form $\{u, \pi\}$. Finally, if $\pi^{\prime} \in \Pi$ is an element with $1+\pi^{\prime} \in U$, we have $-\pi^{\prime} \in \Pi$ and $\left\{1+\pi^{\prime}, \pi\right\}=\left\{1+\pi^{\prime},-\pi^{\prime}\right\}=1$. Hence, $U \cap(1+\Pi)$ is in the kernel. 
The reduction map $\partial: U \rightarrow k_{v}^{*}$ also has $U \cap(1+\Pi)$ in the kernel. Abusing notation, we write $\partial$ for the induced map

$$
\partial: U /\langle U \cap(1+\Pi)\rangle \longrightarrow k_{v}^{*}
$$

where by $\langle U \cap(1+\Pi)\rangle$ we mean the subgroup of $U$ generated by the elements in the set $U \cap(1+\Pi)$. By the previous lemma we have a well-defined and commutative triangle

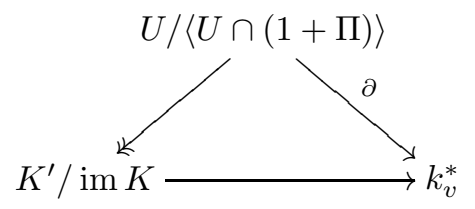

and we see that if $\partial$ is an isomorphism, all maps are isomorphisms. This allows us to divert all attention to the map $\partial$ and to forget about $K$-theory, tame kernels, symbols and even the newly defined $K^{\prime}$ and $K$.

We shall prove that $\partial$ is an isomorphism if $N v$ exceeds a constant depending on $F$. How large we want $N v$ to be gradually becomes clear. At several points in the argument we need a lower bound on $N v$, and we keep track of these bounds by means of boxed assumptions, an example being Assumption 1 above.

In Section 3, we define a set-theoretical map $\gamma: k_{v}^{*} \rightarrow U /\langle U \cap(1+\Pi)\rangle$ for which $\partial \circ \gamma$ is the identity. In Sections 4 and 6 we show that $\gamma \circ \partial$ is the identity on a set of generators for $U /\langle U \cap(1+\Pi)\rangle$. In the course of doing that we find a set of small generators for the group $U_{0}=\left\{x \in U: v^{\prime}(x)=0\right.$ for all $v^{\prime}$ with $\left.N v^{\prime}>d\right\}$ in Section 5. In Section 7 we prove that $\gamma$ is a homomorphism. The definition of $\gamma$ together with the proofs that $\gamma \circ \partial$ is the identity on a set of generators and that $\gamma$ is a homomorphism all rely on assumptions on the norm of $v$. Combining these assumptions, we have proved that $K^{\prime} / \operatorname{im} K \rightarrow k_{v}^{*}$ is an isomorphism. In Section 8 we formulate this as a theorem and deduce the main theorem from it. We also discuss the results for quadratic fields and give asymptotic results.

\section{A CANDIDATE INVERSE MAP}

In this section, we give a candidate inverse map $\gamma$ for $\partial$. In order to define the map, we will make a stronger assumption on the norm of $v$.

In Section 6. we will use balls that are somewhat stretched in one direction and squeezed in another direction. We want the following lemma to be applicable in that situation too, so we need some notation for these stretched and squeezed balls. Let $X$ be the set

$$
X=\left\{\xi \in \prod_{w \in S_{\infty}} \mathbf{R}_{>0}: \prod_{w \in S_{\infty}} \xi_{w}^{n_{w}}=1\right\}, \quad n_{w}=\left[K_{w}: \mathbf{R}\right] .
$$

The set $X$ is a group under pointwise multiplication. By $\xi A$ for $A \subset F_{\mathbf{R}}$ and $\xi \in X$ we mean the set

$$
\xi A=\left\{\left(\xi_{w} a_{w}\right)_{w \in S_{\infty}}: a \in A\right\} .
$$

Multiplication with $\xi$ preserves volumes of measurable sets and norms of elements. Hence, the norm of an element of $\xi B_{t}$ or $\xi C_{t}$ is still at most $t$.

Apart from these squeezed balls we also use cross products of balls. The following lemma relates the packing density of products of balls to the packing densities of balls. For a proof, see [7]. 
Lemma 8 (Hlawka). If $K_{0} \subset \mathbf{R}^{m}$ and $K_{0}^{\prime} \subset \mathbf{R}^{n}$ are bounded symmetric, convex subsets, then the packing density of $K_{0} \times K_{0}^{\prime}$ in $\mathbf{R}^{m+n}$ is at most $\min \left\{\rho\left(K_{0}\right), \rho\left(K_{0}^{\prime}\right)\right\}$.

Lemma 9. If $t \in \mathbf{R}_{>0}$ satisfies $t^{2} \geq \rho d^{2} N v$ and $t<N v$, then for each $u \in k_{v}^{*}$ and $\xi \in X$, there are $x, y \in \xi B_{t} \cap U \cap \mathcal{O}$ with $\partial(x / y)=u$.

Proof. Let $u$ be an element of $k_{v}^{*}$ and let $L$ be the kernel of the map $\mathcal{O} \times \mathcal{O} \rightarrow k_{v}$, given by

$$
(x, y) \longmapsto \bar{x}-u \bar{y} .
$$

The determinant of $L$ is equal to $|\Delta| N v$. Let $t$ satisfy the premises of the lemma. The volume of $\xi B_{t} \times \xi B_{t}$ is equal to $2^{2 n} d^{-2} t^{2}|\Delta|$ and by assumption this is at least $2^{2 n} \rho N v|\Delta|=2^{2 n} \rho \operatorname{det}(L)$. As $\xi B_{t} \times \xi B_{t}$ has packing density at most $\rho$ by Lemma 8 we conclude that there is a nonzero element $(x, y)$ in $\left(\xi B_{t} \times \xi B_{t}\right) \cap L$. The elements $x$ and $y$ have norm smaller than $N v$ and therefore they are either 0 or in $U$. As at least one of them is nonzero, the relation $\bar{x}=u \bar{y}$ tells us that the other element is also nonzero. Hence, they are both in $U$ and we have $\partial(x / y)=u$.

Whenever we have $N v>\rho d^{2}$, we see that $t=\rho^{1 / 2} d(N v)^{1 / 2}$ satisfies the premises of Lemma 9 and hence the map $\partial$ is surjective. We need $N v$ a little bigger in order to ensure that choosing a "random section" gives a well-defined map.

Proposition 10. Suppose $t, t^{\prime} \in \mathbf{R}_{>0}$ satisfy the inequality $2^{n} t t^{\prime}<(N v)^{2}$ and let $\xi$ and $\xi^{\prime}$ be elements of $X$. Assume

$$
x, y \in \xi B_{t} \cap U \cap \mathcal{O}, \quad x^{\prime}, y^{\prime} \in \xi^{\prime} B_{t^{\prime}} \cap U \cap \mathcal{O}
$$

are elements with $\partial(x / y)=\partial\left(x^{\prime} / y^{\prime}\right)$. Then $x / y$ and $x^{\prime} / y^{\prime}$ have the same image in $U /\langle U \cap(1+\Pi)\rangle$.

Proof. It is certainly sufficient to prove that $x y^{\prime}$ and $x^{\prime} y$ have the same image in $U /\langle U \cap(1+\Pi)\rangle$. By Lemma 6, we know that $x y^{\prime}$ and $x^{\prime} y$ are in $\xi \xi^{\prime} D_{t t^{\prime}}$ and we have

$$
x y^{\prime}-x^{\prime} y \in \xi \xi^{\prime} D_{2^{n}} t t^{\prime}
$$

Write $\pi=x y^{\prime}-x^{\prime} y$. It is clear that the valuation of $\pi$ is at least 1 . As we have $N(\pi) \leq 2^{n} t t^{\prime}<(N v)^{2}$, we see that $\pi$ is either 0 or in $\Pi$. When $\pi$ is 0 , the elements $x / y$ and $x^{\prime} / y^{\prime}$ are equal in $U$ and therefore also in $U /\langle U \cap(1+\Pi)\rangle$. When $\pi$ is in $\Pi$, we just write

$$
\frac{x y^{\prime}}{x^{\prime} y}=1+\frac{\pi}{x^{\prime} y} \in 1+\Pi
$$

and we are done.

The existence of a $t$ that satisfies both the requirements in Lemma 9 and the inequality $2^{n} t^{2}<(N v)^{2}$ from Proposition 10] is assured by Assumption 2:

$$
\text { Assumption 2: } N v>2^{n} \rho d^{2} \text {. }
$$

We use this assumption in the definition of $\gamma$.

Definition of $\gamma$. We define a map

$$
\gamma: k_{v}^{*} \longrightarrow U /\langle U \cap(1+\Pi)\rangle
$$

as follows. Let $u$ in $k_{v}^{*}$ be given. Write $t=\rho^{1 / 2} d(N v)^{1 / 2}$. We can find $x, y \in$ $B_{t} \cap U \cap \mathcal{O}$ with $\partial(x / y)=u$ by Lemma 9 . We define

$$
\gamma(u)=\overline{x / y} \in U /\langle U \cap(1+\Pi)\rangle .
$$


By Proposition 10 and Assumption 2, the map $\gamma$ is well defined.

It is clear that $\partial \circ \gamma$ is the identity on $k_{v}^{*}$. In order to prove that $\gamma \circ \partial$ is the identity on $U /\langle U \cap(1+\Pi)\rangle$ we shall use more assumptions.

\section{4. $\gamma \circ \partial$ ON A SET OF GENERATORS (PART 1)}

Recall that $S$ is the set of primes of $F$ such that $U=U_{S}$. Let $v^{\prime}$ be a finite prime in $S$ with

$$
N v^{\prime}>\tilde{d}, \quad \text { with } \tilde{d}=\left(\frac{2}{\pi}\right)^{s}|\Delta|^{1 / 2} .
$$

Let $\mathfrak{p}^{\prime}=\mathfrak{p}_{v^{\prime}}$ be the corresponding prime ideal. The determinant $\operatorname{det}\left(\mathfrak{p}^{\prime-1}\right)$ is equal to $|\Delta|^{1 / 2}\left(N v^{\prime}\right)^{-1}$. Using Minkowski, we see that there exists a nonzero element

$$
\pi^{\prime} \in \mathfrak{p}^{\prime-1} \cap C_{\tilde{d} / N v^{\prime}}
$$

Now $\pi^{\prime} \mathfrak{p}^{\prime}$ is an integral ideal and we have

$$
N\left(\pi^{\prime} \mathfrak{p}^{\prime}\right) \leq\left(\tilde{d} / N v^{\prime}\right) N v^{\prime}=\tilde{d}<N v^{\prime} .
$$

Therefore, the prime ideal factorization of $\left(\pi^{\prime}\right)$ is $\mathfrak{p}^{-1}$ times prime ideals of norm smaller than $N v^{\prime}$. In particular, we have $v^{\prime}\left(\pi^{\prime}\right)=-1$ and $\pi^{\prime} \in U$. Let $\overline{\pi^{\prime}}$ be the image of $\pi^{\prime}$ in $U /\langle U \cap(1+\Pi)\rangle$. We prove that $\gamma \circ \partial$ is the identity on $\overline{\pi^{\prime}}$. Define $t=\rho^{1 / 2} d(N v)^{1 / 2}$ and let $x, y \in B_{t} \cap U \cap \mathcal{O}$ be elements with $\partial\left(\pi^{\prime}\right)=\partial(x / y)$. By Lemma [6] we have

$$
\pi^{\prime} y \in B_{t \tilde{d} / N v^{\prime}} \subset B_{t}
$$

and therefore also

$$
\pi^{\prime} y-x \in B_{2^{n} t} .
$$

This means that the norm of $\pi^{\prime} y-x$ is bounded by $2^{n} t=2^{n} \rho^{1 / 2} d(N v)^{1 / 2}$. We want this to be smaller than $N v$, so we need another assumption:

$$
\text { Assumption 3: } N v>2^{2 n} \rho d^{2} \text {. }
$$

Now we can conclude

$$
N\left(\mathfrak{p}^{\prime}\left(\pi^{\prime} y-x\right)\right)<N v^{\prime} N v \leq(N v)^{2} .
$$

If $\pi^{\prime} y-x$ is nonzero, this tells us that $\pi^{\prime} y-x$ is in $\Pi$. Then we have $\pi^{\prime} /(x / y)=$ $\pi^{\prime} y / x \in 1+\Pi$, which proves $(\gamma \circ \partial)\left(\overline{\pi^{\prime}}\right)=\overline{x / y}=\overline{\pi^{\prime}}$.

Let $U_{0}$ be the group

$$
U_{0}=\left\{x \in U: v^{\prime}(x)=0 \text { for all } v^{\prime} \text { with } N v^{\prime}>\tilde{d}\right\} .
$$

Then $U$ is generated by the set

$$
\left\{\text { all } \pi^{\prime} \text { for all finite } v^{\prime} \in S \text { with } N v^{\prime}>\tilde{d}\right\} \cup U_{0} .
$$

In the next section we find a set of generators for $U_{0}$ and in Section 6 we show that $\gamma \circ \partial$ is the identity on the image of this set in $U /\langle U \cap(1+\Pi)\rangle$. 


\section{FindiNG GOOD GENERATORS FOR $U_{0}$}

Finding "good" generators for $U_{0}$ is almost completely worked out in Section 6 of an article by Lenstra [11]. However, we do have to refer to one of the proofs in this article. While we are at it, we give a refinement of Theorem 6.2 in [11.

Let $T$ be any finite set of primes from $F$, containing $S_{\infty}$ and all finite primes with norm at most $\tilde{d}$. Write $m_{T}=\max \left(\{1\} \cup\left\{N v: v \in T-S_{\infty}\right\}\right)$. According to the proof of Theorem 6.2 in [11, the group $U_{T}$ (which is written there as $K_{S}$ ) is generated by the set

$$
\bigcup_{\substack{\mathfrak{b} \subset \mathcal{O} \\ N \mathfrak{b} \leq \tilde{d}}}\left\{a \in \mathfrak{b}^{-1} \cap U_{T}: \prod_{w \in S_{\infty}} \max \left\{1,\left|a_{w}\right|_{w}^{n_{w}}\right\} \leq \frac{\tilde{d} m_{T}}{N \mathfrak{b}}\right\} .
$$

Although we will not use it, it does not take too much effort to state and prove the next theorem, which is a nice improvement of Theorem 6.2 in [11. Apart from formulation this is identical to Theorem 3 .

Theorem 11. The group $U_{T}$ is generated by the set $C_{\tilde{d}^{2} m_{T}} \cap \mathcal{O} \cap U_{T}$.

Proof. Let $a$ and $\mathfrak{b}$ be as in $(*)$. Write

$$
t=\tilde{d} N \mathfrak{b} \prod_{w \in S_{\infty}} \max \left\{1,\left|a_{w}\right|_{w}^{n_{w}}\right\} .
$$

Then by Minkowski, there is a nonzero $b^{\prime} \in \mathfrak{b}$ with

$$
\left|b_{w}^{\prime}\right|_{w} \leq t^{1 / n} / \max \left\{1,\left|a_{w}\right|_{w}\right\} \quad\left(w \in S_{\infty}\right) .
$$

Because $b^{\prime}$ is in the integral ideal $\mathfrak{b}$ and the norm of $\mathfrak{b}$ is at most $\tilde{d}$, we have $b^{\prime} \in \mathcal{O} \cap U_{T}$. From $a \in \mathfrak{b}^{-1} \cap U_{T}$ it follows that $b^{\prime} a$ is in $b^{\prime}\left(\mathfrak{b}^{-1} \cap U_{T}\right) \subset \mathcal{O} \cap U_{T}$. Because $t$ is at most $\tilde{d}^{2} m_{T}$, we have $b^{\prime}, b^{\prime} a \in C_{\tilde{d}^{2} m_{T}}$. We use these elements as generators instead of $a$. This finishes the proof of the theorem.

If we let $T$ be the set $\left\{\right.$ finite $\left.v^{\prime}: N v^{\prime} \leq \tilde{d}\right\} \cup S_{\infty}$, we have $U_{0}=U_{T}$. The generators we use for $U_{0}$ are the ones given in $(*)$.

\section{6. $\gamma \circ \partial$ ON A SET OF GENERATORS (PART 2)}

By Minkowski, the set $C_{\tilde{d}}$ contains a nonzero element of $\mathcal{O}$ and this implies that $\tilde{d}$ is at least 1 . Hence, in our case we can bound $m_{T}$ by $\tilde{d}$. Let $\mathfrak{b} \subset \mathcal{O}$ be an ideal with $N \mathfrak{b} \leq \tilde{d}$ and let $a \in \mathfrak{b}^{-1}$ be an element with $\prod_{w} \max \left\{1,\left|a_{w}\right|_{w}^{n_{w}}\right\} \leq \tilde{d}^{2} / N \mathfrak{b}$. We prove that $\gamma \circ \partial$ is the identity on the image $\bar{a}$ of $a$ in $U /\langle U \cap(1+\Pi)\rangle$ and we can conclude that $\gamma \circ \partial$ is the identity on the image of a set of generators of $U_{0}$ in $U /\langle U \cap(1+\Pi)\rangle$.

Now is the time we are going to use the $\xi$ 's mentioned in Lemma 9 and Proposition 10 Let $\xi \in X$ be the element such that for $w^{\prime} \in S_{\infty}$ we have

$$
\xi_{w^{\prime}}=\frac{\left(\prod_{w \in S_{\infty}} \max \left\{1,\left|a_{w}\right|_{w}^{n_{w}}\right\}\right)^{1 / n}}{\max \left\{1,\left|a_{w^{\prime}}\right|_{w^{\prime}}\right\}} .
$$

We have chosen $\xi$ in such a way that $a$ is an element of $\xi^{-1} C_{\tilde{d}^{2} / N \mathfrak{b}}$. Define $t=$ $\rho^{1 / 2} d(N v)^{1 / 2}$ and let $x, y \in \xi B_{t} \cap U \cap \mathcal{O}$ be elements with $\partial(x / y)=\partial(a)$ as in Lemma 9] As usual, we want to prove that $a y / x$ is in $\{1\} \cup(1+\Pi)$. It would be 
sufficient if $N(\mathfrak{b}(a y-x))$ is smaller than $(N v)^{2}$. Indeed, in this case $\pi=a y-x$ is either 0 or in $\Pi$ and in the latter case we can write $a y / x=1+\pi / x \in 1+\Pi$.

Clearly, we have

$$
a y \in B_{t \tilde{d}^{2} / N \mathfrak{b}} \text { and } \quad x \in \xi B_{t} \subset B_{t \tilde{d}^{2} / N \mathfrak{b}} \text { and hence } \quad a y-x \in B_{2^{n} t \tilde{d}^{2} / N \mathfrak{b}} \text {. }
$$

Therefore, we have $N(\mathfrak{b}(a y-x)) \leq 2^{n} t \tilde{d}^{2}=2^{n} \rho^{1 / 2} d \tilde{d}^{2}(N v)^{1 / 2}$. For this to be smaller than $(N v)^{2}$, we need Assumption 4:

$$
\text { Assumption 4: } N v>2^{2 n / 3} \rho^{1 / 3}\left(d \tilde{d}^{2}\right)^{2 / 3} \text {. }
$$

\section{When is $\gamma$ A HOMOMORPHism?}

Now that we know that $\gamma \circ \partial$ is the identity on a set of generators - at least, under Assumptions 3 and 4 -and $\partial \circ \gamma$ is the identity everywhere, it suffices to prove that $\gamma$ is a homomorphism. The following lemma is inspired by [1, II.3.2.c].

Lemma 12 (Denominator-trick). If $t \in \mathbf{R}$ satisfies $t^{3} \geq \rho d^{3}(N v)^{2}$ and $t<N v$, then for each $u, u^{\prime} \in k_{v}^{*}$, there are $x, y, z \in B_{t} \cap U \cap \mathcal{O}$ with $\partial(x / z)=u$ and $\partial(y / z)=$ $u^{\prime}$.

Proof. This is almost completely identical to Lemma 9. Let $u$ and $u^{\prime}$ be elements from $k_{v}^{*}$ and let $L$ be the kernel of the map $\mathcal{O} \times \mathcal{O} \times \mathcal{O} \rightarrow k_{v} \times k_{v}$, given by

$$
(x, y, z) \longmapsto\left(\bar{x}-u \bar{z}, \bar{y}-u^{\prime} \bar{z}\right) .
$$

The determinant of $L$ is equal to $|\Delta|^{3 / 2}(N v)^{2}$. The packing density of the cross product of three spheres, each of dimension $n$, is at most $\rho$. Therefore, there is a nonzero element in $(x, y, z)$ in $\left(B_{t} \times B_{t} \times B_{t}\right) \cap L$. The details can easily be adapted from the proof of Lemma 9 ,

The existence of a $t$ as in Lemma 12 is assured by Assumption 5:

$$
\text { Assumption 5: } N v>\rho d^{3} \text {. }
$$

For the next proposition, we also use

$$
\text { Assumption 6: } N v>2^{6 n / 5} \rho d^{12 / 5} \text {. }
$$

Proposition 13. Under Assumptions 2, 5 and 6, the map $\gamma$ is a homomorphism.

Proof. Let $u, u^{\prime}, u^{\prime \prime} \in k_{v}^{*}$ be given with $u u^{\prime}=u^{\prime \prime}$. Write $t=\rho^{1 / 3} d(N v)^{2 / 3}$ and $t^{\prime}=\rho^{1 / 2} d(N v)^{1 / 2}$ and choose $x, y, z \in B_{t} \cap U \cap \mathcal{O}$ according to Lemma 12 and $x^{\prime}, z^{\prime} \in B_{t^{\prime}} \cap U \cap \mathcal{O}$ according to Lemma 9 with

$$
u=\partial(x / z), \quad u^{\prime}=\partial\left(x^{\prime} / z^{\prime}\right), \quad u^{\prime \prime}=\partial(y / z) .
$$

Assumption 6 is chosen precisely to make sure that we have

$$
2^{n} t t^{\prime}<(N v)^{2} .
$$

Hence by Proposition 10, we have $\gamma(u)=\overline{x / z}$ and $\gamma\left(u^{\prime \prime}\right)=\overline{y / z}$ in $U /\langle U \cap(1+\Pi)\rangle$. We want $x x^{\prime}$ and $y z^{\prime}$ to have the same image in $U /\langle U \cap(1+\Pi)\rangle$. As usual, this follows from the fact that $x x^{\prime}-y z^{\prime}$ is in $D_{2^{n} t t^{\prime}}$ and hence, the norm is smaller than $(N v)^{2}$. 
Remark. For specific calculations, the bounds can probably be improved, by not considering all $u^{\prime} \in k_{v}^{*}$ in Proposition [13 but only generators for $k_{v}^{*}$. For many $v$ 's, the group $k_{v}^{*}$ is generated by the images of elements in small balls. To use this, it is probably necessary to use a different ordering on the primes - not necessarily with nondecreasing norms - where the $v$ 's with "large" generators appear later in the ordering.

\section{Discussion}

For the main theorem of this article, we only have to reap what we have sowed. Under Assumptions 1 to 6 , the map $\gamma$ is the inverse map of $\partial$ and as we explained in Section 2, it follows that $K^{\prime} / \mathrm{im} K \rightarrow k_{v}^{*}$ is an isomorphism. We define

$$
c_{F}=\max \left\{2^{2 n} \rho d^{2}, 2^{2 n / 3} \rho^{1 / 3}\left(d \tilde{d}^{2}\right)^{2 / 3}, \rho d^{3}\right\}
$$

and for easy reference, we recall the definitions

$$
d=\frac{2^{n} \Gamma(n / 2+1)}{(\pi n)^{n / 2}}|\Delta|^{1 / 2} \text { and } \tilde{d}=\left(\frac{2}{\pi}\right)^{s}|\Delta|^{1 / 2} .
$$

Furthermore, $\rho$ is the packing density of an $n$-dimensional sphere.

Theorem 14. The map $K^{\prime} / \operatorname{im} K \rightarrow k_{v}^{*}$ is an isomorphism if we have $N v>c_{F}$.

Proof. It suffices to show that Assumptions 1 to 6 follow from $N v>c_{F}$. Assumptions 1 and 2 clearly follow from $N v>2^{2 n} \rho d^{2}$. We see that

$$
2^{2 n} \rho d^{2}, \quad 2^{6 n / 5} \rho d^{12 / 5} \text { and } \rho d^{3}
$$

are equal when we have $d=2^{2 n}$. Hence $2^{6 n / 5} \rho d^{12 / 5}$ is smaller than either $2^{2 n} \rho d^{2}$ or $\rho d^{3}$. Therefore, Assumption 6 follows.

Theorem 15. For every number field $F$, there are constants $c_{F}, c_{F}^{\prime}$ such that

(1) For all $m>c_{F}$, the map $K^{(m)} / \operatorname{im} K^{(m-1)} \rightarrow \bigoplus_{N v=m} k_{v}^{*}$ induced by the maps $t_{v}$ is an isomorphism. The direct sum is taken over all finite primes with norm equal to $m$.

(2) For all $m>c_{F}^{\prime}$, the map $K^{(m)} \rightarrow K^{m}$ is an isomorphism.

For $m>c_{F}$, the image of $K_{2}^{(m)} \mathcal{O}$ in $K_{2} F$ is equal to the tame kernel. For $m>$ $\max \left\{c_{F}, c_{F}^{\prime}\right\}$, the natural map $K_{2}^{(m)} \mathcal{O} \rightarrow K_{2} \mathcal{O}$ is an isomorphism. We can take $c_{F}$ as defined by $(* *)$. The group $K_{2}^{(m)} \mathcal{O}$ is computable as a function of $m$ and $F$.

Proof. Let $m$ be larger than $c_{F}$ and suppose we are in the nontrivial case where there exists a prime with norm equal to $m$. Impose an ordering on the finite primes of $F$ and suppose $w_{1}, w_{2}, \ldots, w_{k}$ are all primes with norm $m$, appearing in this order. Let $v$ be one of the these primes and define $K^{\prime}$ and $K$ as in Section 2 In the case $v=w_{k}$, we have $K^{\prime}=K^{(m)}$ and by Theorem 14, we know that the map $K^{(m)} / \operatorname{im} K \rightarrow k_{v}^{*}$ is an isomorphism. If $v=w_{j}$ for an arbitrary index $j$, we assume $K^{(m)} / \operatorname{im} K^{\prime} \rightarrow \bigoplus_{i=j+1}^{k} k_{w_{i}}^{*}$ is an isomorphism and we use this to show that $K^{(m)} / \operatorname{im} K \rightarrow \bigoplus_{i=j}^{k} k_{w_{i}}^{*}$ is an isomorphism. Consider the diagram below:

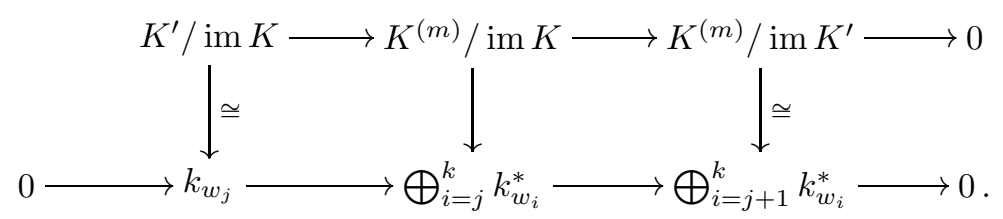


The diagram has exact rows and all maps are compatible. With the snake lemma "without the snake" we see that the middle vertical arrow is in fact an isomorphism. Using induction, we have now proved (1).

As for (2), let $\operatorname{ker}_{m}$ be the kernel of the map $K^{(m)} \rightarrow K^{m}$. Suppose $m>c_{F}$ and consider the commutative and exact diagram

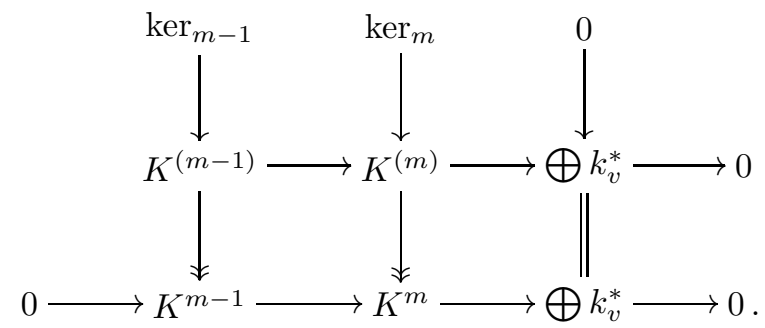

By applying the snake lemma to the diagram, we see that the map $\operatorname{ker}_{m-1} \rightarrow \operatorname{ker}_{m}$ is surjective. In fact, for every $M \geq m$, the map $\operatorname{ker}_{m} \rightarrow \operatorname{ker}_{M}$ is surjective. Because $K_{2} F$ is equal to the direct limit $\lim K^{(m)}$, for each element $x \in$ ker $_{m}$ there is an $M \geq m$ such that $x$ maps to 1 in $\operatorname{ker}_{M}$. Because of the surjectivity of the maps $\operatorname{ker}_{m} \rightarrow \operatorname{ker}_{M}$ and the fact that $\operatorname{ker}_{m}$ is finitely generated, this means there is an $M$ such that $\operatorname{ker}_{M}=0$.

In order to prove that the groups $K_{2}^{(m)} \mathcal{O}$ are computable, we show that we can compute $K^{(m)}$. We can determine $K^{(m)}$, because there are only finitely many solutions to the equation $a+b=1$ with $a, b \in U_{m}$ and we can find them all [3]. Furthermore, factoring out by all elements $a \otimes-a$ for all $a \in U_{m}$ is the same as factoring out by all elements $a \otimes-a$ and $(a \otimes b)(b \otimes a)$ for $a$ and $b$ in a set of generators of $U_{m}$.

It is clear that for $m>c_{F}$, the image of $K_{2}^{(m)} \mathcal{O}$ in $K_{2} F$ is equal to the tame kernel and that for $m>c_{F}^{\prime}$, the map $K_{2}^{(m)} \mathcal{O} \rightarrow K_{2} \mathcal{O}$ is an isomorphism.

It follows from Theorem 15 that the tame kernel is computable if we know its order. We use theorems from Keune to find the order of the $p$-primary part of the tame kernel.

Lemma 16 (Keune). The function $(p, F) \mapsto \#\left(K_{2} \mathcal{O}\right)_{2}$ that maps a prime number $p$ and a number field $F$ to the order of the p-primary part of the tame kernel of $F$ is computable if we either restrict to odd primes or to number fields containing $i$.

Proof. By [8, Theorem 6.6], we can compute the $p$-primary part $W(F)_{p}$ of the wild kernel $W(F)$ when $p$ is an odd prime or $F$ contains $i$, using the isomorphism

$$
\left(\mu_{q} \otimes \mathrm{Cl}\left(\mathcal{O}_{F\left(\zeta_{q}\right)}\left[\frac{1}{p}\right]\right) / q\right)_{\Gamma} \stackrel{\sim}{\longrightarrow} W(F)_{p},
$$

where we have $\Gamma=\operatorname{Gal}\left(F\left(\zeta_{q}\right) / F\right)$ and $q$ is a high enough power of $p$ such that $\mu\left(F_{\mathfrak{p}}\right)_{p} \subset \mu_{q}$ for all $\mathfrak{p} \mid p$ and $q$ kills the $p$-primary part of $K_{2} \mathcal{O}_{F}$. Still for an odd prime $p$, we have a sequence

$$
0 \rightarrow W(F)_{p} \rightarrow\left(K_{2} \mathcal{O}_{F}\right)_{p} \rightarrow \bigoplus_{\mathfrak{p} \mid p} \mu\left(F_{\mathfrak{p}}\right)_{p} \rightarrow \mu(F)_{p} \rightarrow 0
$$

from 8 , (1.10)], which we use to calculate the order of the $p$-primary part of $K_{2} \mathcal{O}_{F}$. All maps are explicitly given in $[8]$. 
Lemma 17. The tame kernel $K_{2} \mathrm{O}$ is computable as a function of number fields $F$ containing $i$.

Proof. By Theorem 15, there exists a value of $m>c_{F}$ such that $K_{2}^{(m)} \mathcal{O}$ is isomorphic to the tame kernel and in particular we can find a value of $m>c_{F}$ such that $K_{2}^{(m)} \mathcal{O}$ is finite. The tame kernel is a quotient of this group and that implies we have an upper bound on the primes occurring in the order. By Lemma 16 we can calculate the order of the tame kernel and by Theorem 15, we can compute the tame kernel.

Lemma 18. The order $\#\left(K_{2} \mathrm{O}\right)_{2}$ of the 2-primary part of the tame kernel is computable as a function of the number field $F$.

Proof. We define $E=F(i)$ and write $\mathcal{O}_{F}$ and $\mathcal{O}_{E}$ for the ring of integers of $F$ and $E$, respectively. By Lemma 17 we can compute $K_{2} \mathcal{O}_{E}$. Let $S$ be the set of infinite primes of $F$ and let $T$ be the set of infinite primes of $E$. Then we can apply [8, Proposition 6.2], which says that the transfer map $K_{2} E \rightarrow K_{2} F$ induces an isomorphism $\left(K_{2}^{+} \mathcal{O}_{E, T}\right)_{\Gamma} \rightarrow K_{2}^{+} \mathcal{O}_{F, S}$, where $\Gamma$ is the Galois group $\operatorname{Gal}(E / F)$. In our case, $K_{2}^{+} \mathcal{O}_{E, T}$ is equal to $K_{2} \mathcal{O}_{E}$ and $K_{2}^{+} \mathcal{O}_{F, S}$ is equal to the kernel of the surjective map $K_{2} \mathcal{O}_{F} \rightarrow \bigoplus_{\mathfrak{p} \text { real infinite }} \mu_{2}$ induced by Hilbert symbols. We can now compute $\left(K_{2}^{+} \mathcal{O}_{F, S}\right)_{2} \cong\left(\left(K_{2} \mathcal{O}_{E}\right)_{\Gamma}\right)_{2}=\left(\left(K_{2} \mathcal{O}_{E}\right)_{2}\right)_{\Gamma}$ and hence we can compute the order of $\left(K_{2} \mathrm{O}_{F}\right)_{2}$.

Theorem 19. The tame kernel is computable as a function of the number field.

Proof. As in the proof of Lemma 17, we can find an upper bound on the primes occurring in the order of the tame kernel. By Lemma 16 and Lemma 18, we can calculate the order of the tame kernel. It follows from Theorem 15 that we can now calculate the tame kernel.

Theorem 20. The smallest feasible value of $c_{F}^{\prime}$ is computable as a function of the number field $F$.

Proof. If for some $m>c_{F}$, the map $K^{(m)} \rightarrow K^{m}$ is an isomorphism, then the map $K^{(m+1)} \rightarrow K^{m+1}$ is also an isomorphism. If, given any $m$, we can check whether the map $K^{(m)} \rightarrow K^{m}$ is injective, or equivalently, whether the map $K^{(m)} \rightarrow K_{2} F$ is injective, it follows that we can find a smallest feasible value of $c_{F}^{\prime}$. We use that we can write $K^{(m)}$ and $K_{2} \mathcal{O}$ with a finite number of generators and relations. First, find explicit generators for the kernel $A$ of the map $K^{(m)} \rightarrow \bigoplus_{v<\infty} k_{v}^{*}$. The images of these generators in $K_{2} F$ are actually in $K_{2} \mathcal{O}$. By a finite search, we can find a representation for these elements in $K_{2} \mathcal{O}$ and we can calculate the kernel of $A$ to $K_{2} \mathrm{O}$ and check if this kernel is 0 in $K^{(m)}$.

Proposition 21. For every number field $F$, we have $c_{F} \leq 4|\Delta|^{3 / 2}$.

Proof. For $n=1$, we have $c_{F}=4$. A numerical approximation shows that the proposition holds for $n=2$. Hence, we assume $n \geq 3$.

Writing $c=2^{n} \Gamma(n / 2+1) /(\pi n)^{n / 2}$, we have $d=c|\Delta|^{1 / 2}$. Using $d \geq 1$ and $\tilde{d} \leq|\Delta|^{1 / 2}$ and $\rho \leq 1$, we get

$$
c_{F} \leq \max \left\{2^{2 n} d^{3}, 2^{2 n / 3} d^{5 / 3} \tilde{d}^{4 / 3}\right\} \leq|\Delta|^{3 / 2} \max \left\{2^{2 n} c^{3}, 2^{2 n / 3} c^{5 / 3}\right\} .
$$

It is enough to prove the inequality $2^{2 n} c^{3} \leq 4$, because that proves that $c$ is smaller than 1 and therefore we have $2^{2 n / 3} c^{5 / 3} \leq\left(2^{2 n} c^{3}\right)^{1 / 3} \leq 4$. Stirling's formula says 
that for every $y>0$ we have $\Gamma(y+1)=\sqrt{2 \pi y}(y / e)^{y} e^{R(y)}$, where $R(y) \leq 1 /(12 y)$ holds (see [9, XIV §64.B]). Hence, using $n \geq 3$, we have

$$
c^{2} \leq n \pi^{1-n} 2^{n} e^{-n+1 / 9} .
$$

It follows that $\log \left(2^{4 n} c^{6}\right)$ is at most $n(7 \log 2-3 \log \pi-3)+3 \log \pi+1 / 3+3 \log n$. A numerical approximation shows that for $n=3$ this is smaller than $\log \left(4^{2}\right)$ and that the derivative is smaller than 0 for $n \geq 3$.

If we specialize to the quadratic case, we have $n=2, s \leq 1, \rho=\pi / \sqrt{12}$. We get the following corollary.

Corollary 22. Let $F$ be a quadratic field with discriminant $\Delta$. Then the $c_{F}$ from Theorem 14 is equal to

$$
c_{F}= \begin{cases}2^{5} 3^{-1 / 2} \pi^{-1}|\Delta|<5.8809|\Delta| & \text { if }|\Delta| \leq 631, \\ 2^{2} 3^{-1 / 2} \pi^{-2}|\Delta|^{3 / 2}<0.2340|\Delta|^{3 / 2} & \text { if }|\Delta|>631 .\end{cases}
$$

Proof. In the quadratic case $2^{2 n / 3} \rho^{1 / 3}\left(d \tilde{d}^{2}\right)^{2 / 3}$ is smaller than $2^{2 n} \rho d^{2}$, no matter if $s$ is 1 or 0 . We have $2^{2 n} \rho d^{2}=\rho d^{3}$ for $|\Delta|=64 \pi^{2} \doteq 631.6$.

The best bounds we have found in the literature are given by Browkin [2]. He proves for imaginary quadratic number fields $F$ that the map $K^{(m)} / \operatorname{im} K^{(m-1)} \rightarrow$ $\bigoplus_{N v=m} k_{v}^{*}$ is an isomorphism when we have $N v>2^{6} \pi^{-10 / 3}|\Delta|^{5 / 3} \doteq 1.41|\Delta|^{5 / 3}$ and $|\Delta| \geq 15$. It is clear that our results are better asymptotically. But also for small discriminants, our bound competes well, as the small table below shows.

\begin{tabular}{lccccccccc}
\cline { 2 - 8 } Discriminant & -15 & -19 & -20 & -23 & $\ldots$ & -148 & -151 & $\ldots$ & -871 \\
Browkin's bound & 128.5 & 190.6 & 207.6 & 262.1 & $\ldots$ & 5836.0 & 6034.5 & $\ldots$ & 111955.1 \\
$c_{F}$ & 88.2 & 111.7 & 117.6 & 135.2 & $\ldots$ & 870.3 & 888.0 & $\ldots$ & 6014.8 \\
\hline
\end{tabular}

The following theorem gives the asymptotic results.

Theorem 23. There are constants $c_{n}$ and $a_{n}$ such that for every number field $F$ of degree $n$ with discriminant $\Delta$, we have $c_{F}<c_{n}|\Delta|^{3 / 2}$ whenever we have $|\Delta| \geq$ $a_{n}$. We can take $c_{n}<\left(2^{-0.599} 2^{3 / 2} e^{-3 / 2} \pi^{-3 / 2}\right)^{n+o(n)}<0.0749^{n+o(n)}$ and $a_{n}<$ $\left(2^{-1} 2^{0.599 \cdot 4 / 3} \pi^{7 / 3} e^{7 / 3}\right)^{n+o(n)}<129.65^{n+o(n)}$ for $n \rightarrow \infty$.

Proof. First of all, the magic "0.599" comes from the Kabatiansky and Levenshtein bound on packing densities of the sphere. They proved that the packing density of an $n$-dimensional sphere is at most $2^{-0.599 n+o(n)}$. This is described in [5, Chapter 9]. An application of Stirling's formula on the bounds in Theorem[14yyields the formulas above.

\section{REFERENCES}

1. H. Bass and J. Tate, The Milnor ring of a global field, Lecture Notes in Mathematics, Vol. 342, pp. 349-446, Springer, Berlin (1973). MR 56:449

2. J. Browkin, Computing the tame kernel of quadratic imaginary fields. With an appendix by Karim Belabas and Herbert Gangl, Mathematics of Computation 69 (2000), no. 232, pp. 1667-1683. MR 2001a:11189]

3. Y. Bugeaud and G. Kálmán, Bounds for the solutions of unit equations, Acta Arithmetica $\mathbf{7 4}$ (1996), no. 1, 67-80. MR 97b:11045

4. H. Cohen, A Course in Computational Algebraic Number Theory, second corrected printing, Graduate Texts in Mathematics, 138, Springer (1995). MR 97e:14001 
5. J. H. Conway and N. J. A. Sloane, Sphere Packings, Lattices and Groups, Grundlehren der mathematischen Wissenschaften, Vol. 290, Springer (1991). MR 93h:11069

6. H. Garland, A finiteness theorem for $K_{2}$ of a number field, Annals of Mathematics (2), 94, (1971), pp. 534-548. MR 45:6785

7. E. Hlawka, Ausfüllung und Überdeckung durch Zylinder, Anzeiger der Österreichischen Akademie der Wissenschaften. Mathematisch-Naturwissenschaftliche Klasse, 85 (1948), nr. 11, pp. 116-119. MR 11:12c

8. F. Keune, On the Structure of the $K_{2}$ of the Ring of Integers in a Number Field, K-Theory 2, (1989), pp. 625-645. MR 90g:11162

9. K. Knopp, Theorie und Anwendung der unendlichen Reihen, Die Grundlehren der mathematischen Wissenschaften in Einzeldarstellungen, Band 2, fünfte Auflage, Springer (1964). MR 10:446a

10. S. Lang, Algebraic Number Theory, second edition, Graduate Texts in Mathematics, 110, Springer (1994). MR 95f:11085

11. H. W. Lenstra, Jr., Algorithms in algebraic number theory, Bulletin of the American Mathematical Society (New Series) 26, (1992), no. 2, pp. 211-244. MR 93g:11131

12. J. Milnor, Introduction to algebraic K-theory, Annals of Mathematics Studies, No. 72, Princeton University Press, Princeton, (1971). MR 50:2304

13. D. Quillen, Higher algebraic K-theory I. In: Algebraic K-Theory I. Lecture Notes in Mathematics, 341, 85-147, Springer (1973). MR 49:2895

14. C. A. Rogers, Packing and covering. Cambridge Tracts in Mathematics and Mathematical Physics, no. 54, Cambridge University Press, New York (1964). MR 30:2405

15. J. Tate, Fourier Analysis in Number Fields and Hecke's Zeta-functions, Thesis Princeton (1950). In: J. W. S. Cassels, A. Fröhlich, Algebraic Number Theory, Thompson, Washington D.C. (1967). MR 36:121

Mathematisch Instituut, Universiteit Leiden, Postbus 9512, 2300 RA Leiden, The NeTHERLANDS

E-mail address: groen@math.leidenuniv.nl

Current address: ABN AMRO, Gustav Mahlerlaan 10, HQ 1056, 1082 PP Amsterdam, The Netherlands 